\title{
Structure and evolution of flux transfer events near dayside magnetic reconnection dissipation region: MMS observations
}

\author{
X.-C. Dong, M. W. Dunlop, K. J. Trattner, \\ T. D. Phan, H.-S. Fu, J.-B. Cao, C. T. Russell, B. L. \\ Giles, R. B. Torbert, Guan Le and J. L. Burch
}

\section{Published version information}

Citation: X Dong et al. "Structure and evolution of flux transfer events near dayside magnetic reconnection dissipation region: MMS observations." Geophysical Research Letters, vol. 44, no. 12 (2017): 5951-5959.

doi: $10.1002 / 2017 G L 073411$

This version is made available in accordance with publisher policies. Please cite only the published version using the reference above. 


\section{Geophysical Research Letters}

\section{RESEARCH LETTER}

10.1002/2017GL073411

\section{Key Points:}

- A series of (three) ion-scale FTEs are observed within $1 \mathrm{~min}$ before MMS crosses the magnetopause reconnection dissipation region

- These FTEs are created by secondary magnetic reconnection and still in the evolution stage after they are ejected from reconnection region

- These FTEs appear to be associated with secondary MR reconnection but have different magnetic field topologies

\section{Correspondence to:}

M. W. Dunlop,

m.w.dunlop@rl.ac.uk

\section{Citation:}

Dong, X.-C., et al. (2017), Structure and evolution of flux transfer events near dayside magnetic reconnection dissipation region: MMS observations, Geophys. Res. Lett., 44, doi:10.1002/ 2017GL073411.

Received 11 MAR 2017 Accepted 2 JUN 2017

Accepted article online 6 JUN 2017

O2017. American Geophysical Union All Rights Reserved.

\section{Structure and evolution of flux transfer events near dayside magnetic reconnection dissipation region: MMS observations}

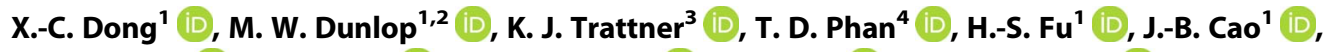 \\ C. T. Russell ${ }^{5}$, B. L. Giles ${ }^{6}$ (D) R. B. Torbert ${ }^{7,8}$ iD, Guan Le ${ }^{6}$ iD, and J. L. Burch ${ }^{8}$ iD \\ ${ }^{1}$ School of Space and Environment, Beihang University, Beijing, China, ${ }^{2}$ RAL_Space, STFC, Chilton, UK, ${ }^{3}$ LASP, University of \\ Colorado Boulder, Boulder, Colorado, USA, ${ }^{4}$ Space Sciences Laboratory, University of California, Berkeley, California, USA, \\ ${ }^{5}$ Department of Earth, Planetary and Space Sciences, University of California, Los Angeles, California, USA, ${ }^{6}$ NASA Goddard \\ Space Flight Center, Greenbelt, Maryland, USA, ${ }^{7}$ Institute for the Study of Earth, Oceans, and Space, University of New \\ Hampshire, Durham, New Hampshire, USA, ${ }^{8}$ Southwest Research Institute, San Antonio, Texas, USA
}

Abstract We investigate a series of three small-scale flux transfer events (FTEs) associated with reconnected flux ropes, recently generated by a nearby, dayside magnetic reconnection line. The data are observed by the Magnetospheric Multiscale spacecraft near noon local time. We find that the associated FTEs are created by secondary magnetic reconnection and have different magnetic field topologies, which is a similar condition to that expected in the multiple X-line magnetic reconnection (MR) model. The calculated results show that the sizes of the FTEs become larger with the time elapsed and the MR reconnection jets at the FTEs are all located on the trailing and outer edges. The above features indicate that these FTEs are still in the evolutionary stage after they are ejected from the reconnection region. Our observations suggest that mesoscale or even typical size FTEs can be created from secondary MR, initially, and subsequently can evolve to a typical size in the process of spreading.

\section{Introduction}

The nature of dayside magnetic reconnection [Dungey, 1961] on the Earth's magnetopause has been extensively investigated with extensive data sets from a number of spacecraft launched and flown over the last few decades, and understanding of the process has progressed significantly. Notably, the fleet of four Cluster spacecraft [Escoubet et al., 1997] has produced multispacecraft measurements, which were able to distinguish spatial structure and evolution to a significant degree for the first time, and had some unique advantages compared with previous single satellite measurements [e.g., Dunlop et al., 1988; Glassmeier et al., 2001; Cao et al., 2006]. The newly launched four Magnetospheric Multiscale (MMS) spacecraft [Burch et al., 2016] are providing multipoint coverage at much smaller spatial scales, and with enhanced plasma measurements, in order to better probe small-scale electron response.

Reconnected flux ropes (FRs) resulting from the unsteady, patchy, or intermittent operation of magnetic reconnection (MR) often manifest themselves as flux transfer events (FTEs), which are typically characterized by a bipolar magnetic field signature in the normal component to the magnetopause [Russell and Elphic, 1978], and typically contain a mixture of magnetosheath-like (MSH) and magnetospheric-like (MSP) plasmas [Paschmann et al., 1982] as a result of newly opened magnetic flux threading the magnetopause (MP). The plasma distributions, both inside and outside the FTEs, depend on where the reconnected FRs are crossed by spacecraft, the time evolution of MR, and the complexity of the reconnection process nearby (such as the presence of secondary reconnection sites [Pu et al., 2013]). Despite extensive studies, the detailed plasma characteristics of FTEs have until recently had limited coverage, so that the generation mechanism still remains unclear. Several models of FTEs have been proposed, for example, the single X-line elbow-shaped model for spatial limited reconnection [Russell and Elphic, 1979], the multiple X-line reconnection model [Lee and Fu, 1985], and the bursty single X-line reconnection model [Scholer, 1988; Southwood et al., 1988]. The FTEs are strictly FRs which thread the MP and therefore have distinct magnetosheath and magnetospheric branches for the first two models and are a bulge of hot plasma for the bursty single X-line reconnection model. The typical sizes of reported FTEs range from fractions of an Earth radii $\left(R_{E}\right)$ to a few $R_{E}$, usually depending on the time elapsed since MR [Fear et al., 2007; Lockwood and Hapgood, 1998; Trattner et al., 2012]. 
Although the influence of magnetic reconnection (MR) takes place over large scales, its onset occurs in small diffusion region centered on the $\mathrm{X}$-line geometry of crossed magnetic fields. Recent kinetic simulations indicated that a small-scale FR can be formed in the diffusion region due to current sheet instabilities as a consequence of large Lundquist number [Daughton et al., 2009; Huang et al., 2009; Lapenta, 2008] or an elongation of the current layers [Daughton et al., 2011; Drake et al., 2006; Guo et al., 2014]. Such small-scale flux ropes have been observed inside or near the reconnection diffusion region in the magnetotail [Chen et al., 2008; Eastwood et al., 2007; Fu et al., 2017; Retinò et al., 2008; Wang et al., 2010], and recently, Eastwood et al. [2016] reported two ion-scale flux rope observations at the magnetopause; although no converging flow and constant size indicating that they were not in the process of growing.

Previous research on FRs and on the resolution of their plasma structure has been somewhat limited by the time resolution of the plasma measurements [Pu et al., 2013; Trenchi et al., 2011, 2016; Zhong et al., 2013]. In comparison to earlier missions such as Time History of Events and Macroscale Interactions during Substorms and Cluster, MMS has a small separation distance and a high-resolution plasma data never achieved before. This unprecedented data enable us to investigate small-scale FRs and details of their plasma structure. Recent notable studies using MMS data have been performed by, e.g., Farrugia et al. [2016], Hwang et al. [2016], Hasegawa et al. [2016], Teh et al. [2017], and Zhao et al. [2016]. In this paper, we analyze four-spacecraft MMS observations of a sequence of three small-scale flux ropes seen just before spacecraft cross the MR diffusion region [Burch and Phan, 2016].

\section{Event Study}

MMS observations taken at Earth's magnetopause on 8 December 2015 are analyzed. The four spacecraft were crossing the magnetopause at a position of $[10.2,1.3,-1.4]$ Earth radius $\left(R_{E}\right)$ in geocentric solarmagnetospheric (GSM) coordinates, where the average separation of the spacecraft was $\sim 10 \mathrm{~km}$. We use Flux-Gate Magnetometer [Russell et al., 2014] and Electron Drift Instrument [Torbert et al., 2015] data for the magnetic field and electric field, as well as Fast Plasma Investigation [Pollock et al., 2016] burst-mode data (150 ms for ion, $30 \mathrm{~ms}$ for electron) for plasma data. All vector data are given in GSM.

Figure 1 shows an overview of the MMS1 observations for the period covering the flux ropes and magnetopause crossing from 11:19:25 UT to 11:21:00 UT. This magnetopause crossing (seen as a $B_{z}$ reversal) near 11:20:43 UT (the last two dashed vertical lines) has been reported previously by Burch and Phan [2016] as an electron dissipation region around the $X$ line. Only GSM is used here as the MP boundary is found to be well aligned in $y, z$, so that the normal direction is close to $x$.

MMS was initially equatorial in the magnetosheath (MSH), which was characterized by negative $B_{z}$ (consistent with a southward IMF), and cold and dense plasma $\left(>10 \mathrm{~cm}^{-3}\right)$. During this interval, three FRs were encountered, marked by pairs of vertical dashed lines corresponding to the intervals 11:19:45 UT to 11:20:04 UT (FR1), 11:20:15 UT to 11:20:23 UT (FR2), and 11:20:32 UT to 11:20:38 UT (FR3). These all contain $B_{x}$ bipolar signatures and an enhanced $|\boldsymbol{B}|$ (Figure 1a), standard characteristics of reconnected FRs. All FRs are seen to have a positive (duskward) $B_{y}$ peak at their center, consistent with either compression of the guide field in the surrounding region, or a Hall magnetic field in the outflow region southward of a site of asymmetric magnetopause reconnection [Tanaka et al., 2008]. Plasma jets exist (mainly in $V_{z}$ ), associated with the flux ropes (FR1 and FR2), with jet speed exceeding $300 \mathrm{~km} / \mathrm{s}$. In the center of the FRs, the total pressure increases, mainly due to the increasing magnetic pressure (Figure 1f), and the parallel ion temperature decreases. The electron temperature (Figure 1e) shows that the electrons are isotropic (equal parallel and perp temperature) in the center but more anisotropic on the two sides of each FR. Because the large core magnetic field of FR1 lies mainly in the first half of the $B_{x}$ bipolar signature, we suggest the spacecraft crossed obliquely through FR1 so that the $B_{x}$ bipolar signature is partly a result of draping of the field lines around the FR. We will therefore just analyze FR2 and FR3 in detail, which exhibit internal structure.

Figures $1 \mathrm{i}$ and $1 \mathrm{j}$ show the Walén scatterplots [Sonnerup et al., 1987; Phan et al., 2004] of $\boldsymbol{V}-\boldsymbol{V}_{\boldsymbol{H} \boldsymbol{T}}$ versus $\boldsymbol{V}_{\boldsymbol{A}}$ for the two time intervals when the spacecraft recorded the jet in the outer edge of FR2 and FR3, respectively, where $\boldsymbol{V}_{\boldsymbol{A}}$ denotes the Alfvén velocity and $\boldsymbol{V}-\boldsymbol{V}_{\boldsymbol{H} \boldsymbol{T}}$ stands for the plasma velocity in the de Hoffmann-Teller (HT) frame [Khrabrov and Sonnerup, 1998]. The slopes of the regression lines are -0.73 and -0.53 , 

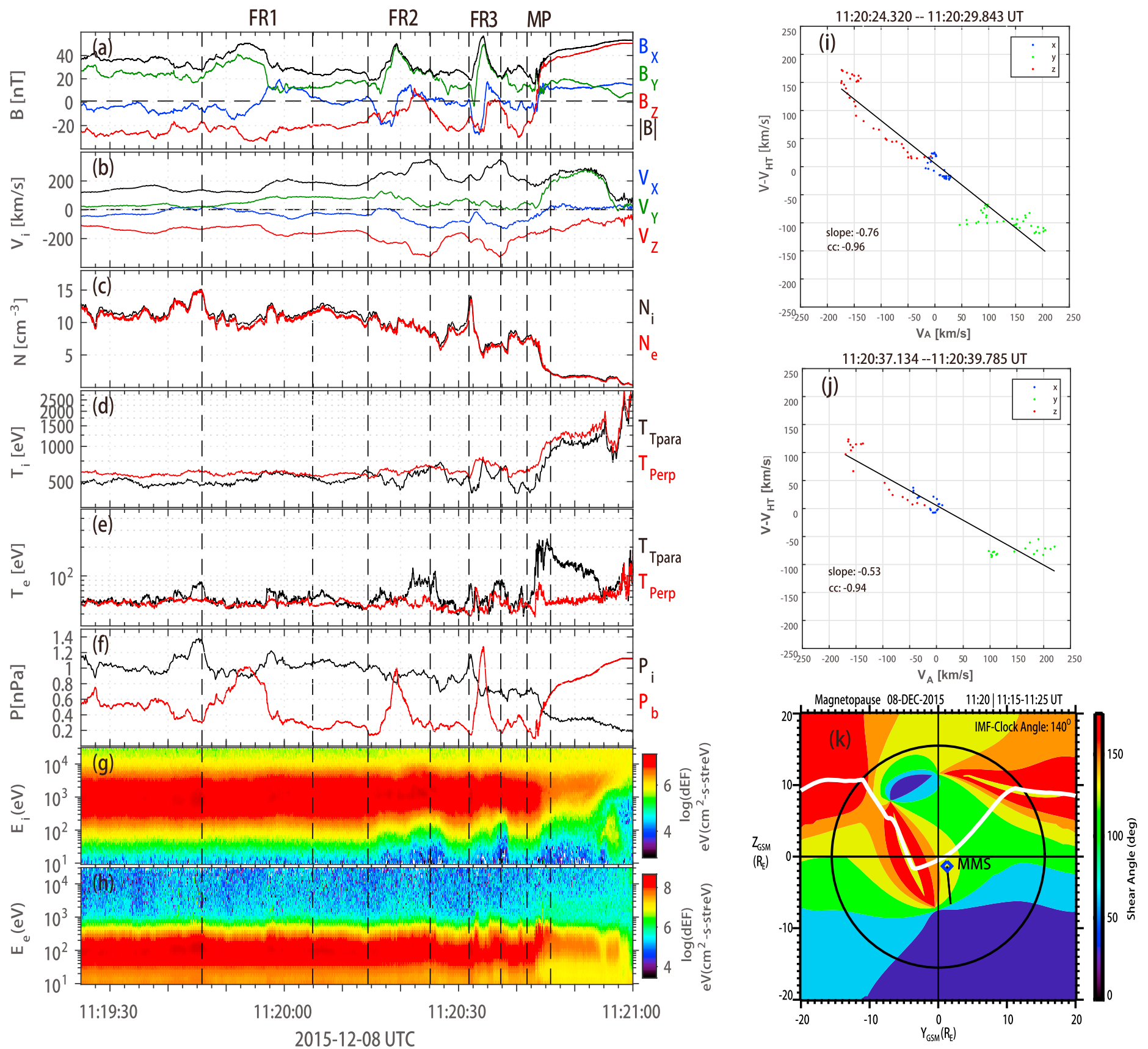

Figure 1. Overview of spacecraft 1 (MMS1) observations showing the positions of the flux ropes and the magnetopause crossing on 8 December 2015. The panels show (a) the vector magnetic field (GSM), (b) the ion velocity (in GSM), (c) the ion and electron densities, (d and e) the ion and electron temperatures, (f) the magnetic and plasma pressures, $(g$ and $h$ ) the electron and ion spectrograms of energy flux, ( $i$ and $j)$ Walén scatterplot for the time interval when MMS recorded the fast jet after FR2 and FR3, and (k) results of the maximum magnetic shear model [Trattner et al., 2007a, 2007b] averaged over 10 min from 11:15 to 11:25 UT. The predicted reconnection location for the solar wind conditions is shown as a thin white line. MMS is located a little south to that of the predicted component magnetic reconnection line.

respectively. Although these slopes are a little far from unity, this is not uncommon in reconnection jetting events [Phan et al., 1996]. Experience has shown that slopes larger than 0.5 can be indicative of a rotational discontinuity (RD) [Paschmann et al., 2005]. The computed values of the correlation coefficients being close to unity indicate a good MP reconnection flow or at least RDs. [Sonnerup et al., 1995; Phan et al., 2004; Vines et al., 2015]. 


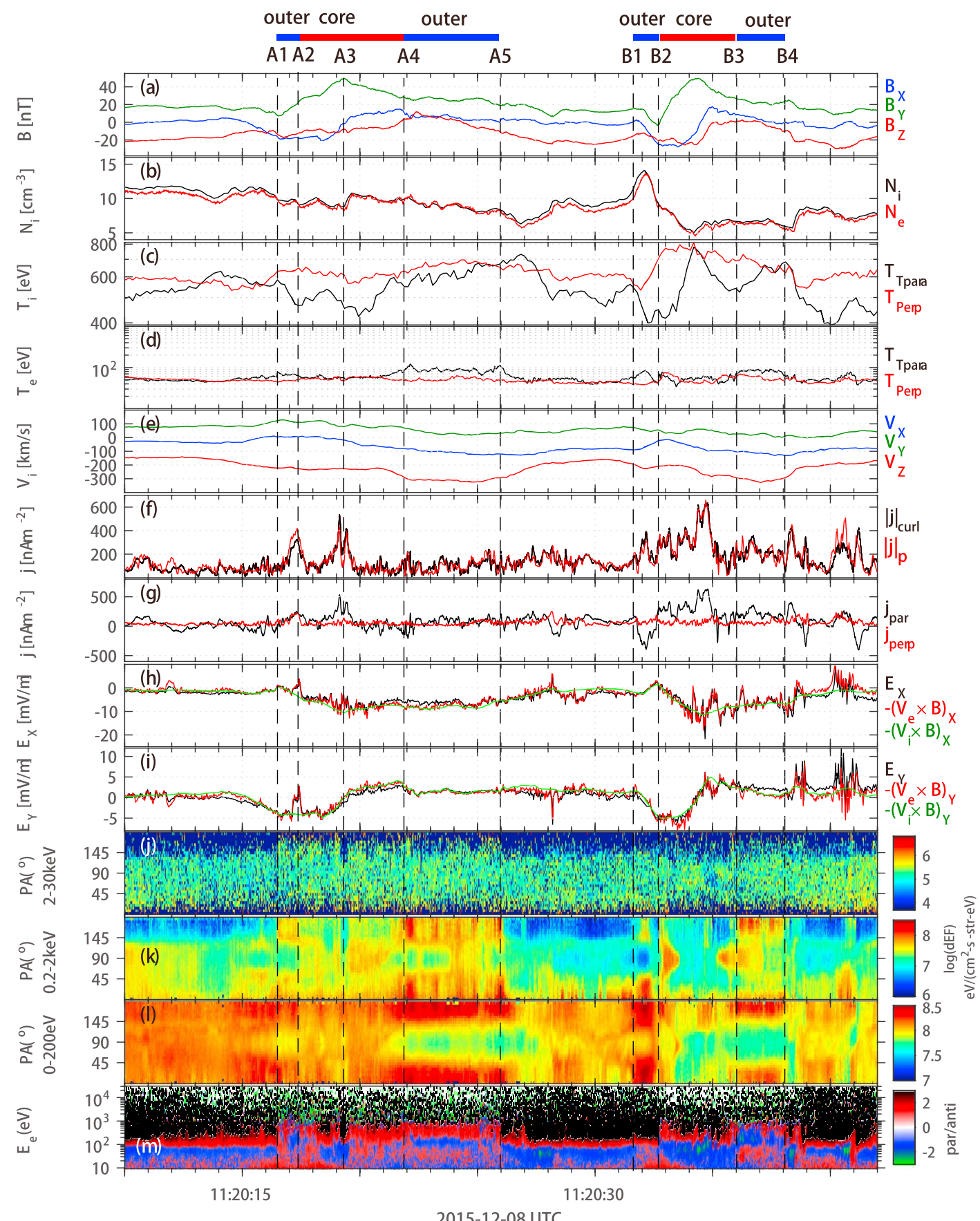

Figure 2. The panels show the (a) magnetic field; (b) ion and electron density; (c and d) ion and electron temperature; (e) ion velocity; ( $\mathrm{f}$ and $\mathrm{g}$ ) electric current density by curlometer and plasma moments; (h and i) measured $E$ (black), $-\boldsymbol{V}_{\boldsymbol{i}} \times \boldsymbol{B}$ (green), and $-\boldsymbol{V}_{\boldsymbol{e}} \times \boldsymbol{B}$ (red) in $x$ and $y$ directions; ( $\left.\mathrm{j}-\mathrm{l}\right)$ the pitch angle distribution of the low-energy $(<200 \mathrm{eV})$, midenergy ( $200 \mathrm{eV}<$ energy $<2 \mathrm{keV}$ ), and high-energy $(2 \mathrm{keV}<$ energy $<30 \mathrm{keV}$ ) electrons; and $(\mathrm{m})$ the electron anisotropy of the parallel/antiparallel energy flux (do negative reciprocal when parallel is large than antiparallel).

\subsection{Motion and Size of FRs}

Figure 2 shows the detailed field and particle signatures from 11:20:10 to 11:20:42 UT, including the intervals for FR2 and FR3. We mark two regions in Figure 2 according to magnetic field and pitch angle distribution: the core region (red) is characterized by an enhanced $B_{y}$ magnetic field, and the outer region (blue) is accompanied by bi-streaming MSH electrons. In the assumed south (leading) boundary of the FR regions, the higher 
Table 1. Results of BMVA and Timing Analysis

\begin{tabular}{lccc} 
& Axis Direction $^{\mathrm{a}}{ }^{(B M V A)}$ & $V_{\mathrm{GSM}}$ in the Core Region (Timing Analysis) & Size of Core Region \\
\hline FR1 & - & $100 \mathrm{~km} / \mathrm{s}^{*}[-0.21,0.66,-0.72]$ & $>1050 \mathrm{~km}\left(\sim 12 d_{i}\right)$ \\
FR2 & {$[-0.40,0.90,-0.15]$} & $169 \mathrm{~km} / \mathrm{s}^{*}[-0.34,0.24,-0.91]$ & $795 \mathrm{~km}\left(\sim 9.0 d_{i}\right)$ \\
FR3 & {$[0.04,0.95,-0.31]$} & $144 \mathrm{~km} / \mathrm{s}^{*}[0.57,-0.08,-0.82]$ & $475 \mathrm{~km}\left(\sim 5.4 d_{i}\right)$ \\
\hline
\end{tabular}

${ }^{\mathrm{a}}$ The axis direction is taken to be the intermediate variance direction of BMVA.

plasma density (Figure 2b) was likely caused by the compression of the FR (most enhanced for FR3). The fast reconnection jets are clearly strongest in the outer region and on the trailing (north) edge of the core region (Figure 2e). This suggests that the flux ropes are pushed by the fast flow similar to observations of Roux et al. [2015] and Trenchi et al. [2016] and gives evidence that reconnection occurred to the north of southward moving FRs, which is consistent with the predicted result of the maximum magnetic shear model [Trattner et al., 2007a, 2007b] (se Figure 1k).

Four spacecraft timing analysis is used here to get the velocity of these FTEs. We use the same method as Eastwood et al. [2016] which treats these flux ropes as cylindrically symmetric and then gets the times when each spacecraft observed the highest magnetic field (Figure 1a) in the core region. The calculated results thus define the orientation of the spacecraft trajectory through the flux ropes in the plane perpendicular to the flux rope axis. Table 1 lists the timing results of the three FRs. The velocities of FR2 and FR3 are found to be mainly in the $x-z$ plane, indicating that the axis of FRs is nearly in the $y$ direction. This orientation is also consistent with the result calculated by magnetic-field-based minimum variance analysis (BMVA) [Sonnerup and Cahill, 1967; Xiao et al., 2004] also shown in Table 1. The size of each FR is calculated by the velocity and durations of their passage (through the core region). It should be mentioned that the spacecraft cross part of the core region of FR1, so the real size of FR1 is larger than $1050 \mathrm{~km}$ (Figure 3). The size of the FR becomes larger from FR3 to FR1, and this is consistent with the distance of the FRs from the reconnection dissipation region, and hence the time elapsed since MR. These results combined with the RD feature and incoming flow seen on one side of FR indicate that these FRs are in the evolutionary stage, after they are ejected from reconnection region. We define these evolutionary FRs as one type of "active" FRs here. Other types of active FRs have been observed. One type corresponds to FRs with two active $X$ lines and converging jets toward the $O$ line [e.g., Øieroset et al., 2011, 2016]; the other type is the "crater" FTE [e.g., Farrugia et al., 2011; Teh et al., 2017; Zhang et al., 2010]. In our case, the evolution is associated with a single $X$ line and is perhaps similar to those seen by Cluster [Phan et al., 2004], which were previously explained by the bursty single X-line reconnection model with a single $\mathrm{X}$-line and modulated reconnection rate, although then for observations far from the $\mathrm{X}$ line. A schematic illustration is presented in Figure 3. We can see that the smallest, FR3, is just $5.4 d_{i}$ (ion inertial length) which corresponds to the ion-scale secondary FR observations of Eastwood et al. [2016], although the FRs in that paper were not active ones.

\subsection{Current and Nonideal Plasma Behavior}

Figure $2 \mathrm{f}$ shows the total current density. The black line is calculated by the curlometer method [Dunlop et al., 2002], and the red line is the average current density at the four-spacecraft barycenter, obtained from plasma velocity moments (red) according to $\boldsymbol{J}=e n_{e}\left(\boldsymbol{v}_{\boldsymbol{i}}-\boldsymbol{v}_{\boldsymbol{e}}\right)$. The agreement between these two estimates serves as

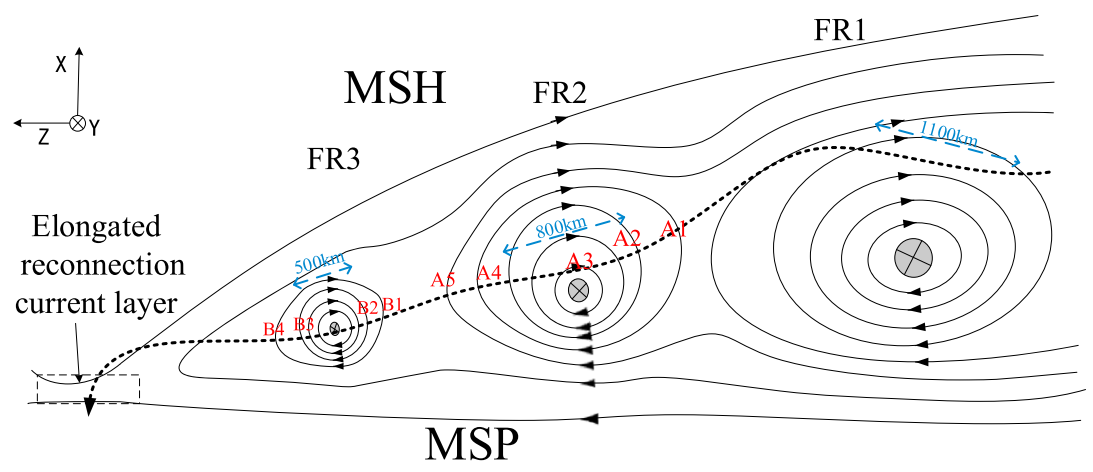

Figure 3. A schematic illustrator of the whole region above and the trajectory of MMS crossing it is shown. 

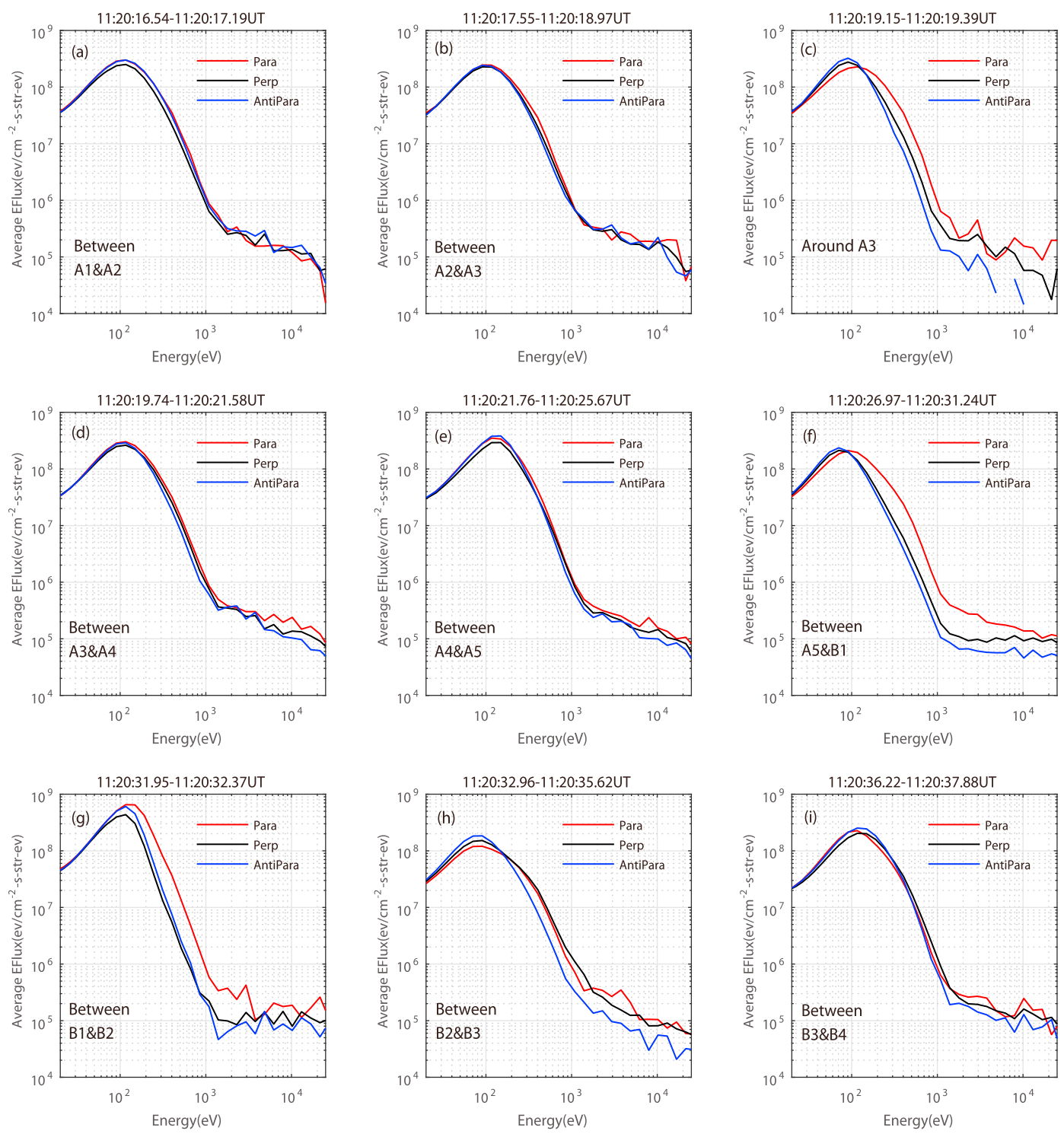

Figure 4. A summary of the typical electron spectra from MMS1 inside the different regions defined in Figure 2. The red, black, and blue lines present the cuts of electron spectra in the parallel, perpendicular, and antiparallel directions.

validation of the current values obtained. The peaks in the current are mainly located in the center and on the (south) boundary of the FRs. The large current in the center can reach to $600 \mathrm{nA} / \mathrm{m}^{-2}$ and shows a clear bifurcated feature in FR2, while the current density is more structured in FR3. Figure $2 \mathrm{~g}$ shows that the electric current is almost parallel to the magnetic field in the center of FRs which means they are predominantly force free. In this case, we can calculate the axis direction of FRs by BMVA [Xiao et al., 2004]: FR2 and FR3 are [ -0.40 , $0.90,-0.15]$ and $[0.04,0.95,-0.31]$ in GSM, respectively, as given in Table 1, near y direction of GSM. This is consistent with the expected direction of two reconnection jets.

Figures $2 \mathrm{~h}$ and 2i compare $E_{x, y}$ with $\boldsymbol{V}_{\boldsymbol{i}} \times \boldsymbol{B}$ and $\boldsymbol{V}_{\boldsymbol{e}} \times \boldsymbol{B}$. We can see that there is agreement between $E_{x, y}$ and $\boldsymbol{V}_{\boldsymbol{e}} \times \boldsymbol{B}$, so $\boldsymbol{V}_{\boldsymbol{e}} \times \boldsymbol{B}$ is dominates the contribution to $E$. The $E_{x, y}$ and $\boldsymbol{V}_{\boldsymbol{i}} \times \boldsymbol{B}$, however, show large deviations which suggest that the ions are not frozen in to magnetic field during those intervals.

\subsection{Electron Distribution and Magnetic Topology}

We discuss the lower plots in Figures $2 j-2 m$, which show the plasma pitch angle (PA) electron distributions in three energy bands and the energy anisotropy, in conjunction with the 1-D electron spectra in Figure 4. In 
Figure 4, we plot the time-integrated electron flux for different intervals (corresponding to the intervals marked through each FTE encounter in Figure 2) as a function of energy, where the distribution is separated according to the parallel $\left(<30^{\circ}\right)$, perpendicular $\left(60^{\circ}-120^{\circ}\right)$, and antiparallel $\left(150^{\circ}-180^{\circ}\right)$ directions. The red, black, and blue lines represent 1-D cuts through electron distribution in the parallel, perpendicular, and antiparallel directions.

For the core region of FR2, the electrons are nearly isotropic for most of the region (Figures $4 \mathrm{~b}$ and $4 \mathrm{~d}$ ) except for a short time around A3 (Figure 4c). The isotropic distribution indicates a closed magnetic field topology. Figure $4 \mathrm{C}$ shows a dominant antiparallel (i.e., incoming) MSH, low-energy electron population ( $<150 \mathrm{eV}$ ), and a dominant parallel (outflowing), heated MSH ( $>200 \mathrm{eV}$ ) and MP (>1000 eV) electrons. This is typical of recently opened magnetic field geometry, connecting to the southern magnetosphere. The PA distributions in Figures $2 \mathrm{k}$ and $2 \mathrm{l}$ for this core interval show a slightly more complex picture, varying through the interval, but nevertheless confirm that broad distributions exist throughout particularly in the low-energy band, with a strong presence of both $0^{\circ}, 90^{\circ}$, and $180^{\circ}$ electrons, consistent with the coincident lines in Figures $4 \mathrm{~b}$ and $4 \mathrm{~d}$. In the outer region of FR2 ( 1 1 and A2 and A4 and A5), the bi-streaming MSH and heated MSH electrons seen in Figures $2 \mathrm{k}-2 \mathrm{~m}$ indicate a trapped (mirrored) electron population, which is dominated by field-aligned populations (also confirmed by Figures $4 \mathrm{a}$ and $4 \mathrm{e}$ which show lower curve for the perpendicular flux). These trapped, high-density electrons may be caused by the magnetic mirror forces created by the curved magnetic field and increasing magnetic strength closer to the flux rope [Hwang et al., 2016]. The MSP electrons ( $>1000 \mathrm{eV}$ ) show a nearly isotropic distribution (see Figures $2 \mathrm{j}$ and $4 \mathrm{a}$ and $4 \mathrm{e}$ ), which indicates closed field line topology also.

In the core region of FR3, the distribution in Figure 4h was characteristic of open topology, showing a similar distribution to that seen in Figure 4c. In this FR the incoming MSH population is clearly seen in the MSH energy band PA distribution (Figure 2l). The MSP energy band PA distribution for this interval, however, shows a significant $90^{\circ}$ distribution, consistent with the coincident parallel and perpendicular lines in Figure $4 \mathrm{~h}$. In the outer region of FR3(B1 and B2 and B3 and B4), the trapped bi-streaming, low-energy and parallel high-energy electrons were characteristics of open field line topology also.

The region between these FRs (Figure $4 f$ ) is typical of recently reconnected magnetic field geometry, connecting to the southern magnetosphere.

Above all, FR2 and FR3 had two kinds of magnetic topology: FR2 was closed for most of the time and open for a small region, while FR3 was almost always open, while also showing a trapped MSP population. This result indicates that a flux rope created by secondary reconnection can be created with more than one kind of field line topology. In multiple X-line reconnection, up to four kinds of topology have been observed [Pu et al., 2013; Zhong et al., 2013]. For secondary reconnection here, this can also be understood because the secondary reconnection also has two reconnection points while remaining in the elongated reconnection current layer.

\section{Summary and Discussions}

The FTE observations analyzed here show the following:

1. A series of (three) ion-scale FTEs are observed within $1 \mathrm{~min}$ before MMS crosses the magnetopause reconnection dissipation region.

2. These FTEs are created by secondary magnetic reconnection and still in the evolution stage after they are ejected from reconnection region (a type of active FTEs; Figure 3).

3. These secondary FTEs have different magnetic field topologies, which is a similar condition to that expected in the multiple $X$-line MR model.

We suggest that the whole sequence may be described as follows:

With a typical guide field the reconnection current layer can become highly elongated. This unstable, elongated current layer can create a flux rope with an enhanced core field to finite amplitude by the process of secondary reconnection and does in this case [Drake et al., 2006]. Subsequently, the flux rope was ejected in the reconnection jet. During the process of spreading downstream, the flux rope was still in the evolution stage, which ultimately develop to a typical size of FTE. 
Thus, our observation suggests that some typical size FTEs [e.g., Phan et al., 2004; Trenchi et al., 2016] which are explained using the bursty single $X$-line reconnection model can possibly be created from secondary MR initially and then evolved to mesoscale or typical size in the process of spreading.

\section{Acknowledgments}

For MMS data visit https://lasp.colorado. edu/mms/sdc/public/. We thank the entire MMS team and instrument PIs for data access and support. M.W.D. is partly supported by an STFC in-house research grant and is supported by the NSFC grants 41574155 and 41431071. This work is partly supported through an ISSI Team "from cluster to MMS." We thank the International Space Science Institute in Bern, Switzerland; its staff; and directors.

\section{References}

Burch, J. L., and T. D. Phan (2016), Magnetic reconnection at the dayside magnetopause: Advances with MMS, Geophys. Res. Lett., 44, 8327-8338, doi:10.1002/2016GL069787.

Burch, J. L., T. E. Moore, R. B. Torbert, and B. L. Giles (2016), Magnetospheric multiscale overview and science objectives, Space Sci. Rev., 199(1-4), 5-21, doi:10.1007/s11214-015-0164-9.

Cao, J. B., et al. (2006), Joint observations by Cluster satellites of bursty bulk flows in the magnetotail, J. Geophys. Res., 111, A04206, doi:10.1029/2005JA011322.

Chen, L., et al. (2008), Observation of energetic electrons within magnetic islands, Nat. Phys., 4(1), 19-23, doi:10.1038/nphys777.

Daughton, W., V. Roytershteyn, B. J. Albright, H. Karimabadi, L. Yin, and K. J. Bowers (2009), Transition from collisional to kinetic regimes in large-scale reconnection layers, Phys. Rev. Lett., 103(6), 1-4, doi:10.1103/PhysRevLett.103.065004.

Daughton, W., V. Roytershteyn, H. Karimabadi, L. Yin, B. J. Albright, B. Bergen, and K. J. Bowers (2011), Role of electron physics in the development of turbulent magnetic reconnection in collisionless plasmas, Nat. Phys., 7(7), 539-542, doi:10.1038/nphys1965.

Drake, J. F., M. Swisdak, K. M. Schoeffler, B. N. Rogers, and S. Kobayashi (2006), Formation of secondary islands during magnetic reconnection, Geophys. Res. Lett., 33, L13105, doi:10.1029/2006GL025957.

Dungey, J. W. (1961), Interplanetary magnetic field and the auroral zones, Phys. Rev. Lett., 6(2), 47-48.

Dunlop, M. W., D. J. Southwood, K.-H. Glassmeier, and F. M. Neubauer (1988), Analysis of multipoint magnetometer data, Adv. Space Res., 8, 273.

Dunlop, M. W., A. Balogh, and K.-H. Glassmeier (2002), Four-point Cluster application of magnetic field analysis tools: The discontinuity analyzer, J. Geophys. Res., 107(A11), 1385, doi:10.1029/2001JA005089.

Eastwood, J. P., T. D. Phan, F. S. Mozer, M. A. Shay, M. Fujimoto, A. Retinò, M. Hesse, A. Balogh, E. A. Lucek, and I. Dandouras (2007), Multi-point observations of the Hall electromagnetic field and secondary island formation during magnetic reconnection, J. Geophys. Res., 112, A06235, doi:10.1029/2006JA012158.

Eastwood, J. P., et al. (2016), lon-scale secondary flux ropes generated by magnetopause reconnection as resolved by MMS, Geophys. Res. Lett., 43, 4716-4724, doi:10.1002/2016GL068747.

Escoubet, C. P., R. Schmidt, and M. L. Goldstein (1997), Cluster-science and mission overview, Space Sci. Rev., 79(1), 11-32, doi:10.1023/ A:1004923124586.

Farrugia, C. J., et al. (2011), "Crater” flux transfer events: Highroad to the X line?, J. Geophys. Res., 116, A02204, doi:10.1029/2010JA015495.

Farrugia, C. J., et al. (2016), Magnetospheric Multiscale Mission observations and non-force free modeling of a flux transfer event immersed in a super-Alfvénic flow, Geophys. Res. Lett., 43, 6070-6077, doi:10.1002/2016GL068758.

Fear, R., S. Milan, A. Fazakerley, C. Owen, T. Asikainen, M. Taylor, E. Lucek, H. Reme, I. Dandouras, and P. Daly (2007), Motion of flux transfer events: A test of the cooling model, Ann. Geophys., 25(1978), 1669-1690, doi:10.5194/angeo-25-1669-2007.

Fu, H. S., A. Vaivads, Y. V. Khotyaintsev, M. André, J. B. Cao, V. Olshevsky, J. P. Eastwood, and A. Retinò (2017), Intermittent energy dissipation by turbulent reconnection, Geophys. Res. Lett., 44, 37-43, doi:10.1002/2016GL071787.

Glassmeier, K.-H., U. Motschmann, M. Dunlop, A. Balogh, C. Carr, G. Musmann, K.-H. Fornacon, K. Schweda, J. Vogt, E. Georgescu, and S. Buchert 2001, CLUSTER as a wave telescope first results from the fluxgate magnetometer, Ann. Geo., Cluster special issue, 19, $1439-1447$.

Guo, F., H. Li, W. Daughton, and Y. H. Liu (2014), Formation of hard power laws in the energetic particle spectra resulting from relativistic magnetic reconnection, Phys. Rev. Lett., 113(15), 155005, doi:10.1103/PhysRevLett.113.155005.

Hasegawa, H., et al. (2016), Decay of mesoscale flux transfer events during quasi-continuous spatially extended reconnection at the magnetopause, Geophys. Res. Lett., 43, 4755-4762, doi:10.1002/2016GL069225.

Huang, Y. M., A. Bhattacharjee, H. Yang, and B. Rogers (2009), Fast reconnection in high-Lundquist-number plasmas due to secondary tearing instabilities, Phys. Plasmas, 16(11), 11,2102-11,2105.

Hwang, K.-J., et al. (2016), The substructure of a flux transfer event observed by the MMS spacecraft, Geophys. Res. Lett., 43, 9434-9443, doi:10.1002/2016GL070934.

Khrabrov, A.V., and B.U.Ö.Sonnerup (1998),DeHofmann-Teller analysis, analysis methods for multi-spacecraft data, edited by G.Paschmann and P.W. Daly, ISSI Sci. Rep., SR-001, 221-248.

Lapenta, G. (2008), Self-feeding turbulent magnetic reconnection on macroscopic scales, Phys. Rev. Lett., 100(23), 3, doi:10.1103/ PhysRevLett.100.235001.

Lee, L. C., and Z. F. Fu (1985), A theory of magnetic flux transfer at the Earth's magnetpause, Geophys. Res. Lett., 12, $105-108$.

Lockwood, M., and M. A. Hapgood (1998), On the cause of a magnetospheric flux transfer event, J. Geophys. Res., 103, 26,453-26,478, doi:10.1029/98JA02244

Øieroset, M., et al. (2011), Direct evidence for a three-dimensional magnetic flux rope flanked by two active magnetic reconnection $\mathrm{X}$ lines at Earth's magnetopause, Phys. Rev. Lett., 107(16), doi:10.1103/PhysRevLett.107.165007.

Øieroset, M., et al. (2016), MMS observations of large guide field symmetric reconnection between colliding reconnection jets at the center of a magnetic flux rope at the magnetopause, Geophys. Res. Lett., 43, 5536-5544, doi:10.1002/2016GL069166.

Paschmann, G., G. Haerendel, I. Papamastorakis, N. Sckopke, S. J. Bame, J. T. Gosling, and C. T. Russell (1982), Plasma and magnetic field characteristics of magnetic flux transfer events, J. Geophys. Res., 87, 2159, doi:10.1029/JA087iA04p02159.

Paschmann, G., S. Haaland, B. U. O. Sonnerup, H. Hasegawa, E. Georgescu, B. Klecker, T. D. Phan, H. Reme, and A. Vaivads (2005), Characteristics of the near-tail dawn magnetopause and boundary layer, Ann. Geophys., 23(4), 1481-1497.

Phan, T. D., G. Paschmann, and B. U. Ö. Sonnerup (1996), Low-latitude dayside magnetopause and boundary layer for high magnetic shear: 2. Occurrence of magnetic reconnection, J. Geophys. Res., 101, 7817-7828, doi:10.1029/95JA03751.

Phan, T. D., et al. (2004), Cluster observations of continuous reconnection at the magnetopause under steady interplanetary magnetic field conditions, Ann. Geophys., 22, 2355-2367.

Pollock, C., et al. (2016), Fast Plasma Investigation for Magnetospheric Multiscale, Space Sci. Rev., 199(1), 1-76, doi:10.1007/s11214-016-0245-4

Pu, Z. Y., J. Raeder, J. Zhong, Y. V. Bogdanova, M. Dunlop, C. J. Xiao, X. G. Wang, and A. Fazakerley (2013), Magnetic topologies of an in vivo FTE observed by Double Star/TC-1 at Earth's magnetopause, Geophys. Res. Lett., 40, 3502-3506, doi:10.1002/grl.50714. 
Retinò, A., et al. (2008), Cluster observations of energetic electrons and electromagnetic fields within a reconnecting thin current sheet in the Earth's magnetotail, J. Geophys. Res., 113, A12215, doi:10.1029/2008JA013511.

Roux, A., P. Robert, D. Fontaine, O. Le Contel, P. Canu, and P. Louarn (2015), What is the nature of magnetosheath FTEs?, J. Geophys. Res. Space Physics, 120, 1-20, doi:10.1002/2015JA020983.

Russell, C. T., and R. C. Elphic (1978), Initial ISEE magnetometer results: magnetopause observations, Space Sci. Rev., 22(6), 681-715, doi:10.1007/BF00212619.

Russell, C. T., and R. C. Elphic (1979), ISEE observations of flux transfer events at the dayside magnetopause, Geophys. Res. Lett., 6, 33-36, doi:10.1029/GL006i001p00033.

Russell, C. T., et al. (2014), The Magnetospheric Multiscale magnetometers, Space Sci. Rev., 199, 189-256, doi:10.1007/s11214-014-0057-3.

Scholer, M. (1988), Magnetic flux transfer at the magnetopause based on single X line bursty reconnection, Geophys. Res. Lett., 15, 291-294, doi:10.1029/GL015i004p00291.

Sonnerup, B. U. Ö., and L. J. Cahill (1967), Magnetopause structure and attitude from Explorer 12 observations, J. Geophys. Res., 72, 171, doi:10.1029/JZ072i001p00171.

Sonnerup, B. U. Ö., I. Papamastorakis, G. Paschmann, and H. Lühr (1987), Magnetopause properties from AMPTE/IRM observations of the convection electric field: Method development, J. Geophys. Res., 92, 12,137-12,159.

Sonnerup, B. U. Ö., G. Paschmann, and T.-D. Phan (1995), Fluid aspects of reconnection at the magnetopause: In situ observations, in Physics of the Magnetopause, Geophys. Monogr. Ser., vol. 90, edited by P. Song, B. U. Ö. Sonnerup, and M. F. Thomsen, pp. 167-180, AGU, Washington, D. C.

Southwood, D. J., C. J. Farrugia, and M. A. Saunders (1988), What are flux transfer events?, Planet. Space Sci., 36(5), 503-508, doi:10.1016/ 0032-0633(88)90109-2.

Tanaka, K. G., et al. (2008), Effects on magnetic reconnection of a density asymmetry across the current sheet, Ann. Geophys., 26(8), 2471-2483, doi:10.5194/angeo-26-2471-2008.

Teh, W.-L., et al. (2017), Evolution of a typical ion-scale magnetic flux rope caused by thermal pressure enhancement, J. Geophys. Res. Space Physics, 122, 2040-2050, doi:10.1002/2016JA023777.

Torbert, R. B., et al. (2015), The electron drift instrument for MMS, Space Sci. Rev., 199(1-4), 283-305, doi:10.1007/s11214-015-0182-7.

Trattner, K. J., J. S. Mulcock, S. M. Petrinec, and S. A. Fuselier (2007a), Location of the reconnection line at the magnetopause during southward IMF conditions, Geophys. Res. Lett., 34, L03108, doi:10.1029/2006GL028397.

Trattner, K. J., J. S. Mulcock, S. M. Petrinec, and S. A. Fuselier (2007b), Probing the boundary between antiparallel and component reconnection during southward interplanetary magnetic field conditions, J. Geophys. Res., 112, A08210, doi:10.1029/2007JA012270.

Trattner, K. J., S. M. Petrinec, S. A. Fuselier, N. Omidi, and D. G. Sibeck (2012), Evidence of multiple reconnectionlines at the magnetopause from cusp observations, J. Geophys. Res., 117, A01213, doi:10.1029/2011JA017080.

Trenchi, L., M. F. Marcucci, H. Rème, C. M. Carr, and J. B. Cao (2011), TC-1 observations of a flux rope: Generation by multiple X line reconnection, J. Geophys. Res., 116, A05202, doi:10.1029/2010JA015986.

Trenchi, L., R. C. Fear, K. Trattner, B. Mihaljcic, and A. N. Fazakerley (2016), A sequence of flux transfer events potentially generated by different generation mechanisms, J. Geophys. Res. Space Physics, 121, 8624-8639, doi:10.1002/2016JA022847.

Vines, S. K., S. A. Fuselier, K. J. Trattner, S. M. Petrinec, and J. F. Drake (2015), Ion acceleration dependence on magnetic shear angle in dayside magnetopause reconnection, J. Geophys. Res. Space Physics, 120, 7255-7269, doi:10.1002/2015JA021464.

Wang, R., Q. Lu, A. Du, and S. Wang (2010), In situ observations of a secondary magnetic island in an ion diffusion region and associated energetic electrons, Phys. Rev. Lett., 104, 175003, doi:10.1103/PhysRevLett.104.175003.

Xiao, C. J., Z. Y. Pu, Z. W. Ma, S. Y. Fu, Z. Y. Huang, and Q. G. Zong (2004), Inferring of flux rope orientation with the minimum variance analysis technique, J. Geophys. Res., 109, A11218, doi:10.1029/2004JA010594.

Zhang, H., et al. (2010), Evidence that crater flux transfer events are initial stages of typical flux transfer events, J. Geophys. Res., 115, A08229, doi:10.1029/2009JA015013.

Zhao, C., et al. (2016), Force balance at the magnetopause determined with MMS: Application to flux transfer events, Geophys. Res. Lett., 43, 11,941-11,947, doi:10.1002/2016GL071568.

Zhong, J., et al. (2013), Three-dimensional magnetic flux rope structure formed by multiple sequential X-line reconnection at the magnetopause, J. Geophys. Res. Space Physics, 118, 1904-1911, doi:10.1002/jgra.50281. 